\title{
ABSTRACTS
}

\section{Cas clinique des experts à l'EBM}

\author{
(C) Springer-Verlag France 2012
}

\section{3}

Cas clinique interactif. Diverticulite : des experts à l'EBM

G. Staumont

Clinique Saint-Jean-Languedoc, 20, route de Revel,

F-31400 Toulouse, France

\section{Mots clés Diverticulite $\bullet$ Recommandations}

L'objectif de ce cas clinique interactif sur la prise en charge des diverticulites est de confronter l'auditoire avec l'avis d'experts ayant rédigé les recommandations françaises publiées en 2007 et l'évolution de l'EBM.

Les définitions ont peu évolué. La diverticulite du côlon correspond à une inflammation ou une infection d'origine diverticulaire. Le terme de sigmoïdite diverticulaire doit être remplacé par le terme de diverticulite sigmoïdienne. La diverticulite compliquée correspond aux complications locales que sont les abcès, les fistules, les péritonites d'origine diverticulaire et les sténoses.

\section{Prévention}

Les moyens de prévenir cette complication sont limités. Chez les patients ayant un antécédent de diverticulite, l'utilisation des corticoïdes et des anti-inflammatoires non stéroïdiens doit être évitée. Par contre, le bénéfice d'une modification de la teneur en fibres dans le régime alimentaire ou d'une décontamination bactérienne n'est pas démontré, tant pour la prévention primaire que secondaire de la diverticulite.

\section{Diagnostic}

Les signes cliniques et biologiques de la diverticulite ne permettent pas seuls une prise en charge thérapeutique adéquate. La tomodensitométrie avec injection de produit de contraste, réalisée dans les 48 heures, est recommandée pour tous les patients cliniquement suspects de diverticulite du côlon, et ce, à chaque épisode afin :

- d'établir le diagnostic positif ;

- de poser un diagnostic alterne ;

- d'aider à la prise de décision thérapeutique en urgence et à distance de la poussée.

L'échographie, examen opérateur dépendant, est moins sensible et moins spécifique que la tomodensitométrie. L'ASP ou le lavement opaque ne sont pas recommandés. La coloscopie, classique ou virtuelle, est contre-indiquée.

\section{Traitement médical d'une diverticulite aiguë}

Une antibiothérapie associant une pénicilline A et un inhibiteur de bêtalactamase ou en cas d'allergie une fluoroquinolone (ofloxacine) associée à un nitro-imidazolé pendant sept à dix jours doit être proposée. L'hospitalisation n'est indiquée qu'en cas de mauvaise tolérance clinique ou en présence de signes de gravité scanographiques. Dans ce cas, une céphalosporine de troisième génération peut être proposée en intraveineux, associée à un nitro-imidazolé.

\section{Traitement chirurgical en urgence en cas de complications}

Le traitement chirurgical en urgence dépend du stade de Hinchey. Les recommandations sont les suivantes :

- dans le stade I (phlegmon ou abcès péricolique) et en cas d'échec du traitement médical et/ou du drainage sous guidage d'imagerie : résection-anastomose, éventuellement protégée par une stomie ;

- dans le stade II (abcès pelvien, abdominal ou rétropéritonéal) : - traitement médical et drainage sous guidage d'imagerie des abcès de plus de $5 \mathrm{~cm}$, puis résection-anastomose sigmoïdienne en un temps à distance ;

- en cas d'échec du drainage, un traitement chirurgical avec résection-anastomose \pm stomie de protection;

- dans le stade III (péritonite généralisée purulente) : résection sigmoïdienne plutôt qu'une stomie de dérivation ;

- dans le stade IV (péritonite fécale) : intervention de référence = intervention de Hartmann (sigmoïdectomie avec fermeture du moignon rectal et colostomie iliaque gauche) ;

- en cas de fistule sigmoïdovésicale : traitement chirurgical, en privilégiant une résection-anastomose en un temps.

\section{Traitement chirurgical prophylactique après une poussée}

Les poussées avec signes de gravité scanographiques sont l'indication d'une chirurgie prophylactique, après un délai d'environ deux mois après la dernière poussée.

En l'absence de signes de gravité scanographiques, le bénéfice réel de la chirurgie prophylactique, même après deux poussées, n'est pas clairement démontré, et il ne doit donc pas être systématique. Chez le sujet de moins de 50 ans, même sans signes de gravité, la chirurgie prophylactique pourrait être proposée dès la première poussée (risque plus élevé de récidive), mais son bénéfice réel est toujours discuté.

La coloscopie avant chirurgie prophylactique est recommandée, en particulier, chez les patients de plus de 50 ans et/ou à risque élevé de cancer rectocolique.

La résection sigmoïdienne prophylactique pour diverticulite doit être réalisée au mieux sous laparoscopie, emportant la charnière rectosigmoïdienne, mais sans extension de la colectomie en amont du sigmoïde même en présence de diverticulose étendue au côlon, car elle ne diminue pas le risque de récidive.

\section{Pour en savoir plus}

Recommandations pour la pratique clinique (2007) Complications de la diverticulose colique. Gastroentérol Clin Biol 31:3S5-3S46 